\title{
Speeding Detection in RFID Systems on Roads
}

\author{
Tao Jing*, Xingni Li*, Wei Cheng ${ }^{\dagger}$, Yan Huo*, Xiaoshuang Xing* \\ ${ }^{*}$ School of Electronics and Information Engineering, Beijing Jiaotong University, China \\ $\dagger$ Department of Computer Science, Virginia Commonwealth University, USA \\ Email: wcheng3@vcu.edu ${ }^{\dagger},\{$ tjing, 12120098, yhou, 11111030\}@bjtu.edu.cn*
}

\begin{abstract}
In this digest paper, we propose a speeding detection system (SDS) based on RFID Systems on Roads (RSR), which consist of RFID tags deployed on road surfaces and RFID readers installed on vehicles. The objective of SDS is to achieve fullroad and all-time speeding detection. The proposed SDS incudes both the speed detection method and the RFID tag deployment method, which is critical to support speeding detection. According to the analytical and the experimental results obtained so far, SDS can successfully detect speeding even if the aggressive drivers are extremely alert.
\end{abstract}

Index Terms-RFID Systems on Roads; speeding detection.

\section{INTRODUCTION}

According to the national traffic accident statistics from Chinese Ministry of Public Security, speeding was the major reason for more than 90 million traffic accidents, which resulted in 7,000 death nationwide in 2012. Currently, speed radars are the most widely utilized devices to detect speeding by the road administration agencies. However, the coverage of speed radars is still very limited due to the high cost of full-road deployment. Although the GPS and speedometer based speeding warning systems have been investigated by the researchers to improve the driving safety in [1-3], the application of these systems for issuing speeding citations is facing multiple challenges: (i) GPS signal is not always available; (ii) Drivers may disable the systems by simply cutting off the power. Another simple speeding detection method is to calculate the average speed of a vehicle between two consecutive toll stations. Obviously, this method will fail if an alter aggressive driver maintains his average speed below the speed limit while speeding in some of the road segments. Note that, highways usually also have low speed limits, but the corresponding detection investigations are still very limited.

Ref. [4] proposed a concept of RFID Systems on Road (RSR), which consist of RFID tags deployed on road surfaces and RFID readers installed on vehicles. In RSR, vehicles can obtain the road information stored in the RFID tags when passing by. Due to the decreasing cost of passive RFID tags, the full-road deployment of RSR is becoming practical. RSR can provide the functions that are missing from the current transportation systems such as lane level localization and navigation, tunnels localization, road distance measurement, and access control. Considering the possible full-road deployment of RSR and its unique essential safety features, which are very attractable to aggressive drivers, we propose to design a full-road and all-time Speeding Detection System (SDS) in this digest paper based on RSR to address all the challenges and issues mentioned above. SDS can detect the speeding violation between two consecutive tags, and it also includes a tag deployment method for maximizing the detection accuracy while reducing the cost of deploying RFID tags. Note that SDS will be part of the RSR system. Thus, a driver cannot solely deactivate SDS (e.g. by cutting off power) while still using the service provided by other RSR applications. The major contributions of this research can be summarized as follows.

- We propose SDS that can detect all-road full-time speeding based on RSR.

- We analyze the behavior of an extreme alert aggressive driver who knows the system setup and tries to escape from being detected when speeding.

- We design a RFID tag deployment method to assist SDS to deal with extreme alert and aggressive drivers.

- Simulations demonstrate that SDS can successfully detect speeding with an appropriate tag deployment.

\section{ARChitecture OF SDS}

SDS is designed based on the RSR system as shown in Fig. 1, where the distance between two consecutive tag is $D$, and the vehicle reads the $i$ th $\operatorname{tag}\left(\operatorname{Tag}_{i}\right)$ at time $t_{i}, 0 \leq i \leq 4$.

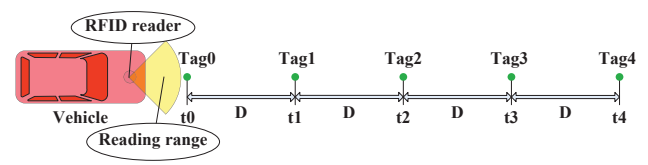

Fig. 1. The Architecture of SDS

The vehicle records the time $t_{i}$ when passing by the tag $\mathrm{Tag}_{i}$. As $D$ can be obtained through calculating the distance between the locations of two tags, we can have the average speed between tags $\mathrm{Tag}_{i}$ and $\mathrm{Tag}_{i+1}$ as follows.

$$
\bar{v}_{i}=\frac{D}{t_{i+1}-t_{i}}
$$

The vehicle can also obtain the road speed limit $v_{\text {limit }}$ through reading the RFID tags. If the average speed between two tags is larger than $v_{\text {limit }}$, SDS will record this violation and send it to the road administrative agency via V2V or V2I communications. SDS will also notify the neighbor vehicles with the existence of speeding vehicles so that the drivers are not caught of guard.

If the distance $D$ is too large, an alert and aggressive driver, who is aware of the tag deployment strategy, can adjust his speed so that his average speed is below the speed limit while 
speeding in some road segments. On the contrary, if $D$ is too small, the deployment cost will be increased largely. In the following, we therefore focus on finding an appropriate $D$ so that SDS can catch extremely alert and aggressive drivers while reducing the deployment cost via analysing the drivers' behaviors.

\section{The Analysis of Alert Driver's Behavior}

According to the statistics in [5], the average acceleration of cars is about $4 \mathrm{~m} / \mathrm{s}^{2}$, and the maximum acceleration is between $6 \mathrm{~m} / \mathrm{s}^{2}$ and $7 \mathrm{~m} / \mathrm{s}^{2}$. The research on the relationship between acceleration and a passenger's comfortability [6] shows that the passenger feels comfortable and uncomfortable when the acceleration is $\leq 1.8 \mathrm{~m} / \mathrm{s}^{2}$ and $\geq 5 \mathrm{~m} / \mathrm{s}^{2}$, respectively. The emergent deceleration of cars is between $9 \mathrm{~m} / \mathrm{s}^{2}$ and $10 \mathrm{~m} / \mathrm{s}^{2}$ according to the statistics in [7]. Conservatively, we set the acceleration and the deceleration of an alert and aggressive driver as $6.5 \mathrm{~m} / \mathrm{s}^{2}$ and $10 \mathrm{~m} / \mathrm{s}^{2}$, respectively, so that our study can cover even the worst cases.

To avoid being detected by SDS, an alert and aggressive drive may drive the car within two tags by following the three procedures below.

1) Acceleration: The driver accelerates the car from its initial speed $v_{0}$ (the speed of the vehicle when reading the first tag) to $v_{\max }$ with the acceleration at $6.5 \mathrm{~m} / \mathrm{s}^{2}$.

2) Change Point: The driver depresses the brake pedal to decelerate. According to the general performance of cars, it takes $0.2 \mathrm{~s}$ to gradually reach the maximal deceleration $\left(10 \mathrm{~m} / \mathrm{s}^{2}\right)$.

3) Deceleration: The driver decelerates the car to a speed $v_{\text {end }}$ with the deceleration at $10 \mathrm{~m} / \mathrm{s}^{2}$ so that the average speed during the time of the three procedures is below $v_{\text {limit }}$.

Note that we assume that the time between any of the two consecutive procedures is zero so that the above procedures represent the best that a driver can do to avoid being caught by SDS while reaching the speed of $v_{\max }$.

\section{RFID TAG DEPLOYMENT}

If $v_{\max }, v_{\text {limit }}$, and $v_{0}$ have been given, we, therefore, can solve the $D$, the minimal tag distance required to catch a driver if his top speed is $v_{\max }$ on a road with speed limit $v_{\text {limit }}$, from the equations below.

$$
\begin{aligned}
S_{1}+S_{2}+S_{3} & =D \\
S_{1} & =\left(v_{\max }^{2}-v_{0}^{2}\right) /\left(2 \times a_{1}\right) \\
a_{2} & =a_{3} / t_{2} \\
v_{t_{2}} & =v_{\max }-\int_{0}^{t_{2}} a_{2} \times t d t \\
S_{2} & =\int_{0}^{t_{2}}\left(v_{\max }-a_{2} \times t\right) d t \\
S_{3} & =\left(v_{t_{2}}^{2}-v_{\text {end }}^{2}\right) /\left(2 \times a_{3}\right) \\
t_{1} & =\left(v_{\max }-v_{0}\right) /\left(a_{1}\right) \\
t_{2} & =0.2 \text { second }
\end{aligned}
$$

$$
\begin{aligned}
t_{3} & =\left(v_{t_{2}}-v_{\text {end }}\right) /\left(a_{3}\right) \\
v_{\text {limit }} & =D /\left(t_{1}+t_{2}+t_{3}\right)
\end{aligned}
$$

, where $S_{1}, S_{2}$, and $S_{3}$ are the distances that the vehicle travels during procedures of acceleration, change point, and deceleration, respectively. $v_{t_{2}}$ is the vehicle's speed after the second procedure. $a_{1}=6.5 \mathrm{~m} / \mathrm{s}^{2}, a_{2}$, and $a_{3}=10 \mathrm{~m} / \mathrm{s}^{2}$ are the acceleration during the procedures of acceleration, change point, and deceleration, respectively. $t_{1}, t_{2}$, and $t_{3}$ are the times that the vehicle stays in each procedure.

Similarly, given $D, v_{\text {limit }}$, and $v_{0}$, we can also obtain the $v_{\max }$ that a driver can achieve while without being caught when strictly following the three procedures through solving the equations.

\section{Simulations}

We use MATLAB to evaluate the performance and the deployment requirement of SDS. We set that a driver will be issued a citation if the average speed of his vehicle is larger than $v_{\text {limit }}$. We set $v_{\max }=110 \% \times v_{\text {limit }}$ to calculate the minimal $D$ for several road segments, which have different speed limit, for catching the drivers whose average speed between two consecutive tags are larger than $v_{\max }$. Note that all the other parameters are set as mentioned above. The table reports the simulation results for the $D$ required under several typical scenarios.

\begin{tabular}{|c|c|c|c|c|}
\hline$v_{\text {limit }}=$ & $30 \mathrm{~km} / \mathrm{h}$ & $60 \mathrm{~km} / \mathrm{h}$ & $100 \mathrm{~km} / \mathrm{h}$ & $120 \mathrm{~km} / \mathrm{h}$ \\
\hline$v_{0}=0.9 \times v_{\text {limit }}$ & $4.6 \mathrm{~m}$ & $16.8 \mathrm{~m}$ & $44.0 \mathrm{~m}$ & $61.7 \mathrm{~m}$ \\
\hline$v_{0}=v_{\text {limit }}$ & $3.9 \mathrm{~m}$ & $13.9 \mathrm{~m}$ & $36.1 \mathrm{~m}$ & $50.6 \mathrm{~m}$ \\
\hline$v_{0}=1.1 \times v_{\text {limit }}$ & $2.6 \mathrm{~m}$ & $8.4 \mathrm{~m}$ & $20.4 \mathrm{~m}$ & $28.0 \mathrm{~m}$ \\
\hline
\end{tabular}

Based on the results above, the simulations demonstrate that the minimal distance $D$ should be $2.6 \mathrm{~m}, 8.4 \mathrm{~m}, 20.4 \mathrm{~m}$, and $28.0 \mathrm{~m}$ for the road speed limit at $30 \mathrm{~km} / \mathrm{h}, 60 \mathrm{~km} / \mathrm{h}, 100 \mathrm{~km} / \mathrm{h}$, and $120 \mathrm{~km} / \mathrm{h}$, respectively. It is intuitive to observe that the tag distance $D$ decreases when the initial speed increases.

More evaluation results (which cannot be presented here due to the page limit) justify that SDS can successfully detecting speeding and issue citations when the speed is larger than $v_{\max }$ with an appropriate tag deployment distance.

\section{FUTURE WORKS}

In the future, we will implement SDS on a RSR test-bed.

\section{REFERENCES}

[1] P. Wilson, D. Prashanth, and H. Aghajan, "Utilizing rfid signaling scheme for localization of stationary objects and speed estimation of mobile objects," in RFID, 2007. IEEE International Conference on. IEEE, 2007, pp. 94-99.

[2] J. Pérez, F. Seco, V. Milanés, A. Jiménez, J. C. Díaz, and T. De Pedro, "An rfid-based intelligent vehicle speed controller using active traffic signals," Sensors, vol. 10, no. 6, pp. 5872-5887, 2010.

[3] H. Yang and S.-H. Yang, "Rfid based automatic speed limit warning system," in Control 2010, UKACC International Conference on. IET, 2010, pp. 1-6.

[4] W. Cheng, X. Cheng, M. Song, B. Chen, and W. Zhao, "On the design and deployment of rfid assisted navigation systems for vanets," Parallel and Distributed Systems, IEEE Transactions on, vol. 23, no. 7, pp. 1267-1274, 2012.

[5] XGO. The vehicle acceleration rankings. [Online]. Available: http : //labs.xgo.com.cn/top 4. html

[6] B. Ye, "Plus the acceleration of the material point," Mechanics in Engineering, vol. 10, no. 5, pp. 51-53, 1988.

[7] XGO. The vehicle braking rankings. [Online]. Available: http : //labs.xgo.com.cn/top thtml $_{5}$ 